\title{
Kernos
}

Revue internationale et pluridisciplinaire de religion grecque antique

$24 \mid 2011$

Varia

\section{Adrienne DIMAKOPOULOU, Chlôrêis aêdôn pâle rossignol. Une étude sémantique}

\section{Maud Clerfays}

\section{OpenEdition \\ Journals}

\section{Édition électronique}

URL : http://journals.openedition.org/kernos/1992

DOI : 10.4000/kernos. 1992

ISSN : 2034-7871

\section{Éditeur}

Centre international d'étude de la religion grecque antique

\section{Édition imprimée}

Date de publication : 1 janvier 2011

Pagination : 358-359

ISSN : 0776-3824

\section{Référence électronique}

Maud Clerfays, "Adrienne dimakopoulou, Chlôrêis aêdôn pâle rossignol. Une étude sémantique », Kernos [En ligne], 24 | 2011, mis en ligne le 18 octobre 2011, consulté le 21 septembre 2020. URL : http:// journals.openedition.org/kernos/1992 ; DOI : https://doi.org/10.4000/kernos.1992 


\section{Adrienne Dimakopoulou, Chlôrêis aêdôn pâle rossignol. Une étude sémantique, Paris, Apolis Éditions, 2010. 1 vol. 14,8 × 21 cm, 156 p. ISBN : 978-2-9532495-3-8.}

Ce livre, paru en août 2010, est en fait la publication d'un travail de fin d'études réalisé, il y a une trentaine d'années, sur le mythe antique du Rossignol raconté par Pénélope au chant XIX de l'Odyssée. Dès lors, on pourrait s'attendre à lire une version remaniée et complétée par une nouvelle bibliographie qui comporterait notamment des références postérieures. Mais il n'en est rien car les éditions Apolis ont pris le parti de publier uniquement le texte initial.

Tout au long de l'Antiquité, l'image du rossignol a souvent été associée aux femmes légendaires en proie à la douleur, mais pourquoi ? Est-ce que l'expression signifie seulement qu'elles pleurent? Au cœur de l'interrogation se trouve le sens de l'adjectif contenu dans l'énoncé Chlôrêis aêdôn, mais sans l'enfermer dans son sens premier, à savoir chromatique, car « quand on a dit 'vert' qu'a-t-on dit?» (p. 80). L'A. prend le parti d'explorer tout d'abord les catégories mentales et affectives impliquées par l'adjectif chlôros, en étudiant l'association de cet adjectif à certains termes du champ sémantique de la peur, à savoir chlôron deos et chlôron deima. À partir de passages d'Homère et des Suppliantes d'Eschyle, elle éclaire le sens de chlôros en analysant les circonstances qui sont à l'origine des deux craintes dont il est fait mention dans les textes : d'une part, la méconnaissance du danger (Ulysse et son désir de vengeance) et d'autre part la souillure (Io, ce mélange monstrueux de deux natures). Dès lors, elle choisit de traduire l'adjectif chlôros par «trouble» et introduit, tout en finesse, le chiffre deux du doute en faisant remarquer que l'aspect hybride de Io n'est pas la seule cause du trouble car sa présence dans un lieu sacré, comme celle de Déméter au palais de Célée et Métanire, l'est tout autant. Elle précise ensuite son propos en abordant la descente aux Enfers d'Ulysse. Dans cet univers, le héros est lui aussi saisi par la "crainte trouble ». En effet, il ressent la chlôron deos dès qu'il voit tous ces morts sans mémoire qui poussent des cris «terrifiants », et il la ressentira encore lorsqu’il sera fait mention de Gorgô. L'A. s'arrête aussi sur la pratique du béros et, partant de l'accent mis sur la transformation d'Ulysse en aède par Fr. Frontisi-Ducroux, elle remarque que ce statut lui permet d'éviter le lot commun des mortels ordinaires qui consiste à mourir dans la mémoire populaire. Enfin, pour clore cette approche de la Nekyia, elle se penche sur la valeur étymologique de deos. Elle pointe ainsi du doigt le lien entre la racine indo-européenne $*$ dwei qui donne deos et le thème numéral *dwei, « deux ». À ce moment précis, l'A. côtoie à nouveau le doute qui mène à l'hésitation entre deux possibilités. Mais l'enquête est loin d'être terminée. La question : «Aédon est-elle chlôra de peur ou de printemps comme la végétation ? (p. 51), lui offre la possibilité de passer progressivement du champ sémantique de la peur à celui des larmes. Elle note d'emblée que si Homère associe très souvent l'adjectif thaleros (florissant) aux larmes, Sophocle, lui, le remplace par chlôros (l'appartenance de ces deux signifiants à l'univers végétal permettant de passer de l'un à l'autre sans aucune difficulté). Il ne s'agit pas de n'importe quelles larmes mais bien des dakrua chlôra: des larmes de femmes bien sûr, car ce sont toujours les femmes qui ont peur et qui pleurent vite, comme si le chlôron n'avait en fait déteint que sur elles. La vingtaine de pages consacrée aux "Larmes troubles des femmes» (p. 55-75), ces larmes de Déjanire, d'Hélène et de Médée, trois femmes qui pleurent les morts dues à leur ignorance, soutient les propos antérieurs de l'A. à savoir que si la peur est provoquée par la méconnaissance, le trouble, lui, l'est par le doute et par l'obscurité. Fidèle à sa méthode, elle poursuit son enquête en analysant, le temps d'une nouvelle parenthèse, le terme adinos. Ce passage qui fait ressortir toutes les difficultés liées à la transposition des valeurs sémantiques d'une langue à une autre, l'amène à conclure qu'adinos semble, quand on parle de larmes, finalement fort proche du sens de chlôros. Le poème «À une aimée » de Sappho, lui permet ensuite d'aborder le domaine de l'éros, en approchant plus particulièrement un type de désir amoureux qui est chlôron comme Sappho est chlôrotera poias. La lecture de ces vers constitue une douce transition qu'utilise l'A. pour s'engager dans l'étude de la valeur chromatique de 
chlôros. En abordant le sens premier de chlôros, elle rappelle qu'en grec ancien, il est rare qu'un signifiant désigne par lui-même une couleur. Ainsi chlôros renverrait plutôt à une nuance en référence au monde de la végétation naissante. De plus, elle rappelle que «dans des civilisations différentes, on ne voit pas de la même façon!». Il faut dès lors chercher dans les textes comment les Grecs eux-mêmes définissaient la nature des couleurs. Platon et Aristote en parlent notamment en employant le terme chroia qui signifie, outre la couleur, la surface, notion de perception visuelle des limites. Pour eux, posséder une couleur, c'est être délimité, être doté d'une forme. Démocrite a aussi livré une théorie des couleurs. Selon lui, le vert est la seule à ne pas posséder de morphê mais à être constituée de plein et de vide. Ce type de mélange est pourtant commun à toutes les couleurs et ne peut dès lors définir le seul vert. La nature de la couleur verte ne transgresserait-elle pas simplement les lois de la théorie de Démocrite, couleur «trouble » qui ne peut que brouiller les limites ? L'A. poursuit son voyage dans le domaine des couleurs en mettant cette fois l'accent sur le terme pepsis utilisé pour rendre compte des diverses colorations. En effet, les couleurs évoluent jusqu'à l'accomplissement de ce qu'elles colorent. L'A. remet cette affirmation en relation avec ses résultats antérieurs sur la mort et le statut héroïque pour aboutir à l'idée d'une mort crue qui se différencierait de la mort pourpre mise en évidence par Gernet. Le livre se referme sur deux versions du mythe du Rossignol et de l'Hirondelle en langue originale avec traduction, ainsi que sur un index.

Cet intéressant petit livre propose une étude sémantique fondée sur une méthode personnelle, novatrice et rigoureuse. Il permet de mieux cerner certaines représentations mentales et culturelles proprement grecques. L'A. nous emmène dans un voyage riche de ses réflexions aux tournures poétiques, fait remarquable lorsqu'on sait que le français n'est pas sa langue maternelle. Cependant, il n'est pas toujours aisé de suivre l'A. dans les méandres de sa pensée car non seulement les relations entre les divers paragraphes ne sont pas forcément claires, mais aussi le choix d'une langue métaphorique ne facilite pas toujours la compréhension. Enfin, le monde des couleurs en Grèce a connu un regain d'intérêt ces dernières années et la confrontation des conclusions du présent ouvrage et de ces recherches nouvelles s'imposera à ceux qu'intéressent ces questions.

Maud Clerfays (Université de Liège)

Corinne Bonnet, Vinciane Pirenne-Delforge, Danny Praet (éds), Les religions orientales dans le monde grec et romain: cent ans après Cumont (1906-2006). Bilan historique et historiographique. Colloque de Rome, 16-18 novembre 2006, Bruxelles/Rome, Institut Historique Belge de Rome, 2009. 1 vol. $19 \times 25,5$ cm, 464 p. (Institut Historique Belge de Rome. Études de philologie, d'archéologie et d'histoire anciennes, 45). ISBN : 978-90-74461-71-9.

Pour célébrer le centième anniversaire de la publication de la première édition de l'ouvrage de Franz Cumont, Les religions orientales dans le paganisme romain ${ }^{1}$, issu de conférences qu'il avait faites au Collège de France en 1905, un colloque a été organisé à Rome en novembre 2006. Son objet principal était de proposer une réflexion sur l'actualité et la pertinence de ce thème. Le volume d'actes que voici compte vingt-quatre contributions, réparties en cinq sections. Une étude introductive, due aux éditeurs, présente brièvement les axes de recherche sur lesquels reposent les réflexions de cette rencontre. Une attention particulière y est accordée à la validité de la notion de «cultes orientaux », une catégorie entrée dans l'usage, mais qui se révèle en fait

$14^{\mathrm{e}}$ édition, Paris, 1929, dont il existe à présent une réédition par les soins de Corinne Bonnet et de Françoise Van Haeperen, Turin, 2006 (Bibliotheca Cumontiana. Scripta Maiora, 1). 